\title{
Dynamics of curved reaction fronts under a single-equation model
}

\author{
$\begin{array}{ll}\text { R. P. Bhanot } & \text { D. V. Strunin } \\ & \end{array}$
}

(Received 8 January 2016; revised 11 February 2018)

\begin{abstract}
Fronts of reaction in certain systems (such as so-called solid flames) are modelled by a high-order nonlinear partial differential equation, which we analyse numerically. Previously, Strunin [IMA J. Appl. Math. 63:163-177, 1999] obtained stable spinning solutions of the equation using the Galerkin method. Here we use a more sophisticated and arguably more powerful method, namely the one-dimensional radial basis function method, to study the equation further. As an initial step, we elaborate the numerical code and tested it by reproducing the spinning regimes for a range of initial conditions. In a new series of experiments, we find a regime where the front is shaped as a pair of kinks spinning in a stable joint formation. The settled character of this regime is demonstrated.
\end{abstract}

DOI:10.21914/anziamj.v57i0.10446 gives this article, (c) Austral. Mathematical Soc. 2018. Published March 20, 2018, as part of the Proceedings of the 12th Biennial Engineering Mathematics and Applications Conference. ISSN 1445-8810. (Print two pages per sheet of paper.) Copies of this article must not be made otherwise available on the internet; instead link directly to the DOI for this article. 


\section{Contents}

1 Introduction

C399

2 The numerical method

C401

3 Numerical experiments

C402

3.1 Travelling front under homogeneous boundary conditions . C403

3.2 Travelling front under periodic boundary conditions . . . . C404

4 Conclusions

C408

References

C410

\section{Introduction}

Certain types of active systems with dissipation satisfy

$$
\partial_{t} u=-A\left(\partial_{x} u\right)^{2} \partial_{x}^{2} u+B\left(\partial_{x} u\right)^{4}+C \partial_{\chi}^{6} u,
$$

where $A, B$ and $C$ are constants $(A>0, C>0)$. In particular, equation (1) is relevant to combustion waves (fronts) [1] and nonlinear instabilities in reaction-diffusion systems [2]. In the context of combustion waves, $\mathfrak{u}$ stands for the distance passed by the combustion front along a hollow cylinder, $x$ is a running coordinate along the circumference of the cylinder and $t$ is time. Equation (1) generates a rich variety of dynamics, the most spectacular of which is the spinning wave illustrated in Figure 1. Figure 1 shows periodic solutions of (1) at different times on the surface of a cylinder, rolled out into a plane (two periods are shown).

Equation (1) was solved in 1D with one independent spatial dimension $x$ using the spectral Galerkin method [1], and in 2D using a finite difference scheme [3]. In this paper we apply a different numerical method, namely 
Figure 1: A spinning front solution of equation (1) derived by Strunin [1].

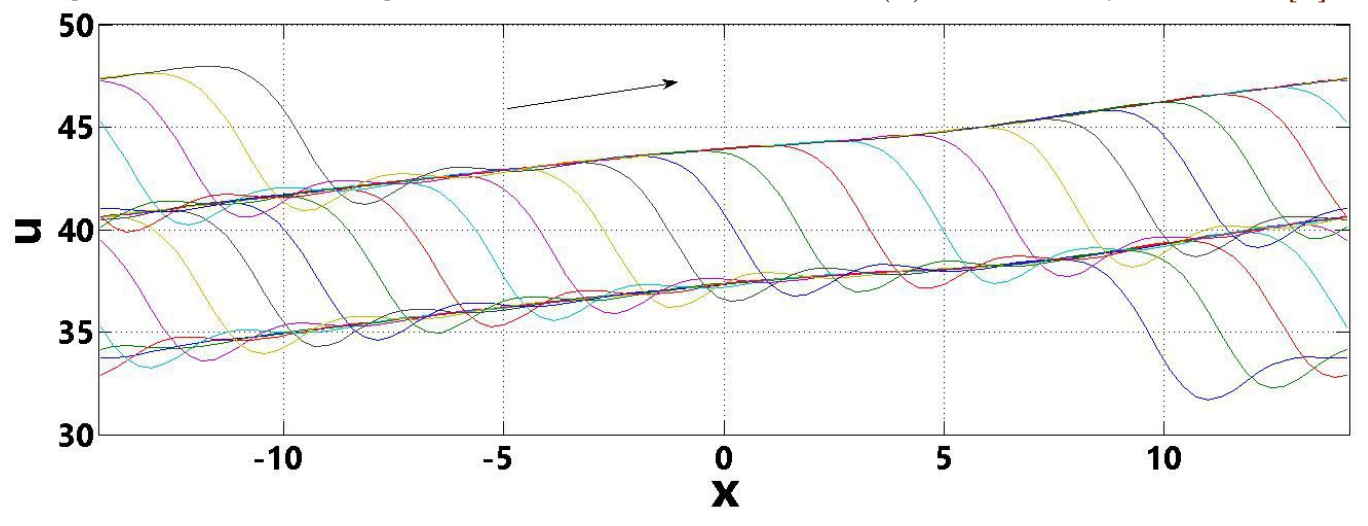

the 1D integrated radial basis function networks method (1D-IRBFN), which has proved to be efficient in a number of applied problems [13, 14]. Firstly we obtain a spinning wave solution and compare it with Figure 1. In this figure we see a train of fronts, each comprising a steep section (step) followed by a long, nearly flat plateau. The plateau appears inclined, but the slope is small. The speed of the fronts, as well as their height and width, are controlled by the equation and not initial conditions. This step-like structure is a consequence of the balance between the energy release, represented by the term $-A\left(\partial_{x} u\right)^{2} \partial_{x}^{2} \mathfrak{u}$, and the dissipation, represented by the term $C \partial_{x}^{6} \mathfrak{u}$. The term $B\left(\partial_{x} u\right)^{4}$ plays the role of a bridge between the two, as explained by Strunin $[1,2,3]$. By re-scaling $\boldsymbol{t}, \boldsymbol{x}$ and $\boldsymbol{u}$, equation (1) can always be transformed into canonical form where all the coefficients, $A, B$ and $C$ become unity.

\section{The numerical method}

The 1D-IRBF and IRBF-based methods were successfully verified through several engineering problems such as turbulent flows [4], laminar viscous 
flows [5, 6, 7], structural analysis [8], and fluid-structure interaction [9].

Radial basis function networks (RBFNs) are known as powerful high-order approximation tools for scattered data [10]. A function $\boldsymbol{f}(\boldsymbol{x})$, to be approximated, is represented by an RBFN as

$$
f(\boldsymbol{x}) \approx u(x)=\sum_{i=1}^{N} w_{i} G_{i}(\boldsymbol{x}),
$$

where $\boldsymbol{x}$ is the input vector, $\mathrm{N}$ is the number of RBFs, $\left\{\boldsymbol{w}_{i}\right\}_{i=1}^{\mathrm{N}}$ is the set of network weights to be found, and $\left\{G_{i}(\boldsymbol{x})\right\}_{i=1}^{\mathrm{N}}$ is the set of RBFs. According to Micchelli's theorem [20], there is a large class of RBFs, for example the multiquadric, inverse multiquadric and Gaussian functions, whose design/ interpolation matrices obtained from (2) are always invertible. It was proved that RBFNs are capable of representing any continuous function to a prescribed degree of accuracy [20]. Furthermore, according to the Cover theorem [20], the higher the number of RBFs used, the more accurate the approximation will be, indicating the property of 'mesh convergence' of RBFns. Among RBFs, the multiquadric functions, $G_{i}(\boldsymbol{x})=\sqrt{\left(\boldsymbol{x}-\mathbf{c}_{i}\right)^{\top}\left(\boldsymbol{x}-\mathbf{c}_{i}\right)+a_{i}^{2}}$, with $\boldsymbol{c}_{i}$ the centre and $\boldsymbol{a}_{\boldsymbol{i}}$ the width, are ranked as the most accurate and possess an exponential convergence with the spatial discretisation refinement [20]. The application of RBFNs for solving partial differential equations has received wide attention over the last decades [11, e.g., and references therein]. The usual approach is to differentiate (2) as often as required to obtain approximate derivatives of $f(\boldsymbol{x})$ [12]. If the error in $f(\boldsymbol{x})$ is $O\left(h^{s}\right)$, where $h$ is the mesh size and $s>0$, then the error in the $n$th derivative of $f(x)$ is $O\left(h^{s-n}\right)$. In other words, there is a reduction in the convergence rate for derivatives and this reduction is an increasing function of derivative order. Thus, differentiation will magnify any error that might exist in the approximation of $\boldsymbol{f}(\boldsymbol{x})$.

To avoid the convergence rate decreasing with differentiation, and recognising that integration is a smoothing process, the integral formulation was proposed $[13,14]$, where spectral approximants (e.g. RBFNs) are utilised to represent the highest-order derivatives under consideration and then integrated analytically to yield approximate expressions for lower-order derivatives and 
the function itself. This approach is called integrated radial basis function networks (IRBFN). Although RBF methods can be easily implemented in a truly meshless manner based on scattered data points, it proves very efficient and effective to discretise a domain using Cartesian grids [13, 14]. Thus, the purpose of using integration (a smoothing operator) to construct the approximants is to avoid the reduction in convergence rate caused by differentiation, and also to improve the numerical stability of the discrete solution.

The integration process naturally gives rise to arbitrary constants that serve as additional expansion coefficients, and therefore facilitate the employment of some extra equations in the process of converting the RBF weights into the function values. This distinguishing feature of the integral formulation provides effective ways to overcome well-known difficulties associated with conventional differential approaches: (i) the implementation of multiple boundary conditions [15]; (ii) the description of non-rectangular boundaries on a Cartesian grid [16]; (iii) the imposition of high-order continuity of the approximate solution across subdomain interfaces [17]; and (iv) the incorporation of nodal derivative values into the approximations via compact IRBFN stencils (C-IRBFN) [18].

The ability of the IRBFN methods to capture very sharp gradients, which is highly desirable for equation (1), was demonstrated with the effective simulation of shockwave-like behaviour in the dynamic strain localisation of a quasi-brittle material subjected to a sudden step loading [19]. 


\section{Numerical experiments}

\subsection{Travelling front under homogeneous boundary conditions}

In this set of numerical experiments we assume the homogeneous boundary conditions

$$
\begin{aligned}
& \left.\partial_{\chi} u\right|_{x=L_{1}}=\left.\partial_{\chi} u\right|_{x=L_{2}}=0, \\
& \left.\partial_{\chi}^{2} u\right|_{x=L_{1}}=\left.\partial_{\chi}^{2} u\right|_{x=L_{2}}=0, \\
& \left.\partial_{\chi}^{3} u\right|_{x=L_{1}}=\left.\partial_{\chi}^{3} u\right|_{x=L_{2}}=0 .
\end{aligned}
$$

In this context, $\mathrm{L}$ is the perimeter of the cylinder and $\mathrm{L}_{1} \leqslant \mathrm{~L} \leqslant \mathrm{~L}_{2}$. The equation coefficients were chosen arbitrarily, $A=2, B=1, C=1$. We recall that, regardless of concrete values of the coefficients, the governing equation can always be transformed to the canonical form with $\mathrm{A}=\mathrm{B}=\mathrm{C}=1$ by re-scaling $x, t$ and $u$. The space domain is constantly shifted to the right, following the moving front. The number of nodes is 100, and the covered length is $L_{2}-L_{1}=30$. The initial condition is

$$
u(x, 0)=5 \exp \left[-(x-1)^{2}\right] .
$$

After some transitional period (Figure 2), the front clearly settles into a constant shape (most evident at the later times), with nearly horizontal tails (Figure 3). Ahead of the front is a characteristic chain of small-scale oscillations caused by the high-order dissipation. Effectively, the kink in Figure 2 is almost the same as an individual kink from the train in Figure 1 because Figure 2 presents the limiting case of Figure 1 when the spatial period goes to infinity (the period in Figure 1 is already very large compared to the kink's width). In the next section we present a more direct matching between our results and the periodic solution in Figure 1. 
Figure 2: The front at early times $t=2$ (top) and $t=3$ (bottom).
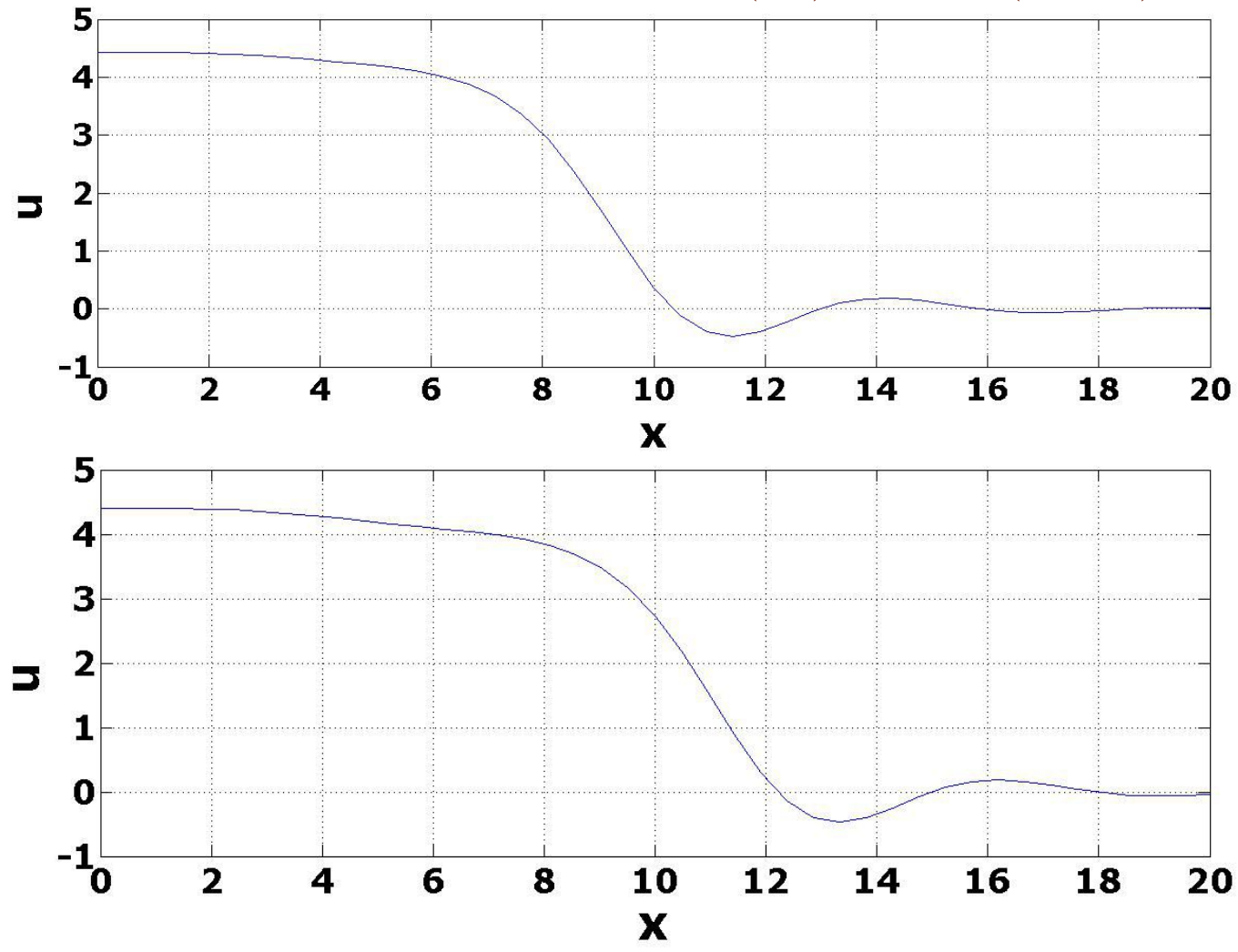

\subsection{Travelling front under periodic boundary conditions}

For these numerical experiments we imposed periodic boundary conditions

$$
\begin{aligned}
& \left.u\right|_{x=L_{1}}=\left.u\right|_{x=L_{2}},\left.\quad \partial_{\chi} u\right|_{x=L_{1}}=\left.\partial_{\chi} u\right|_{x=L_{2}}, \\
& \left.\partial_{\chi}^{2} u\right|_{x=L_{1}}=\left.\partial_{\chi}^{2} u\right|_{x=L_{2}},\left.\quad \partial_{\chi}^{3} u\right|_{x=L_{1}}=\left.\partial_{\chi}^{3} u\right|_{x=L_{2}} \text {, } \\
& \left.\partial_{\chi}^{4} u\right|_{x=L_{1}}=\left.\partial_{\chi}^{4} u\right|_{x=L_{2}},\left.\quad \partial_{\chi}^{5} u\right|_{x=L_{1}}=\left.\partial_{\chi}^{5} u\right|_{x=L_{2}} \text {. }
\end{aligned}
$$


Figure 3: Settling of the fixed-shape front.

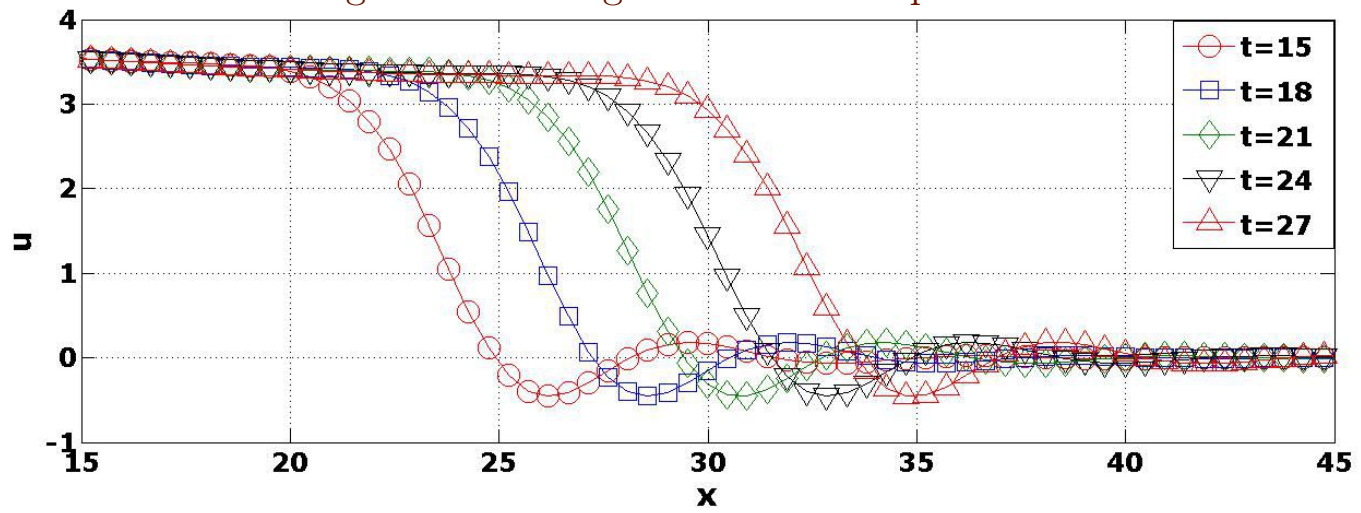

The solutions obtained in this section are associated with the reaction front moving on a cylindrical surface. In this context, $L$ is the perimeter of the cylinder and $\mathrm{L}_{1} \leqslant \mathrm{~L} \leqslant \mathrm{~L}_{2}$.

In the following numerical experiment we used $A=8, B=3$ and $C=2$ and the lumpy initial condition

$$
u(x, 0)=2 \sin x .
$$

Over one period $\mathrm{L}_{2}-\mathrm{L}_{1}=4 \pi$ the initial profile (4) performs four oscillations, as seen in Figure 4. The number of nodes is 100, and the time step is 0.001 . Figures 5 and 6 show the early evolution of the front where the initially symmetric shape gradually becomes asymmetric. We explain this effect by the small initial asymmetry introduced by spatial discretisation. Over time the asymmetry amplifies, with the four initial crests gradually merging into one. Figure 7 presents the later stages of the evolution during which a singlestep regime is eventually established. Thus, the small initial asymmetry progresses into a fully developed step-like formation travelling to the left. This numerical experiment again demonstrates independence of the settled regime (to be precise, the hight and width of individual steps, but not their number) to the details of the initial condition. 
Figure 4: The initial condition (4).

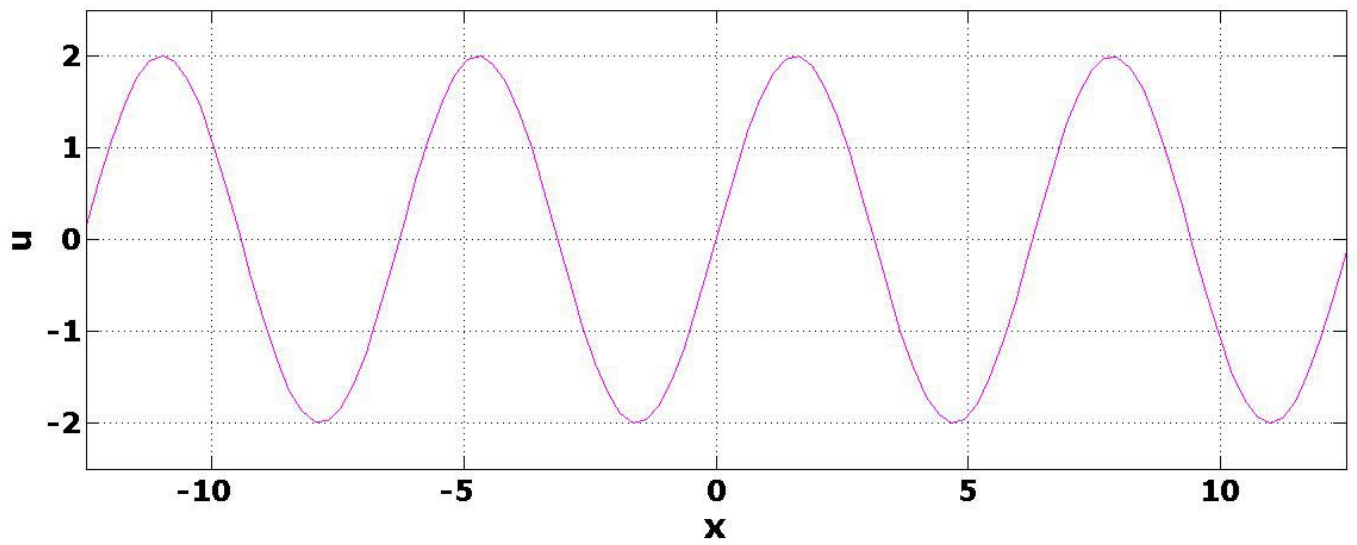

Figure 5: The solution evolved from (4) between $t=2.1$ and $t=2.9$.

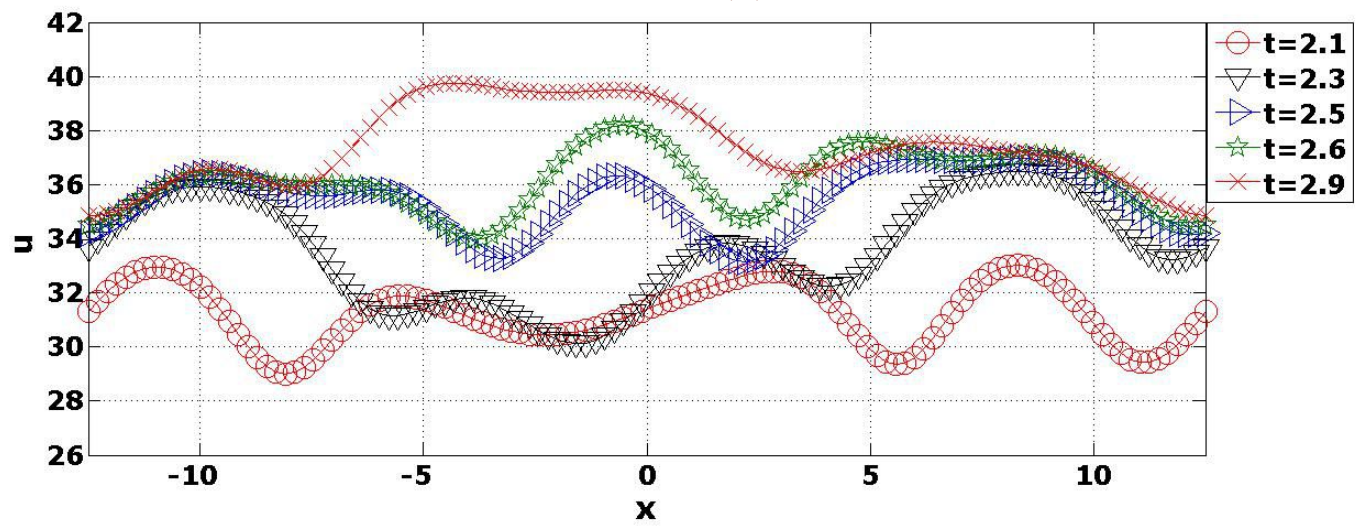


Figure 6: The solution between $t=4.9$ and $t=6.5$.

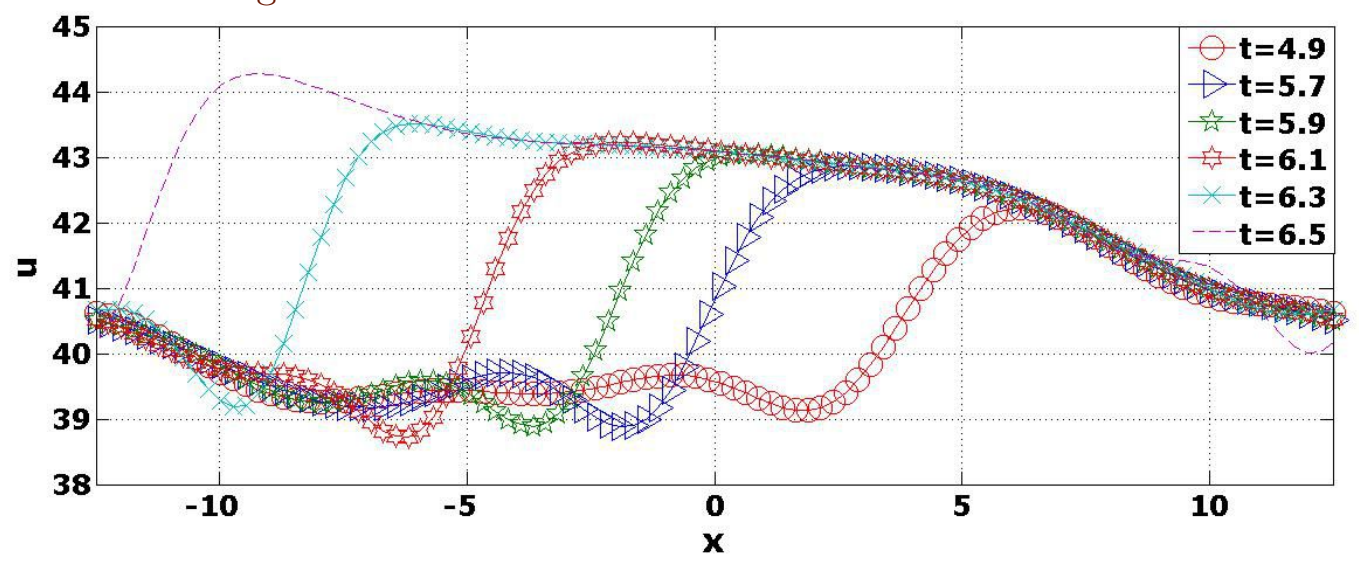

Figure 7: The solution between $t=6.8$ and $t=9.6$.

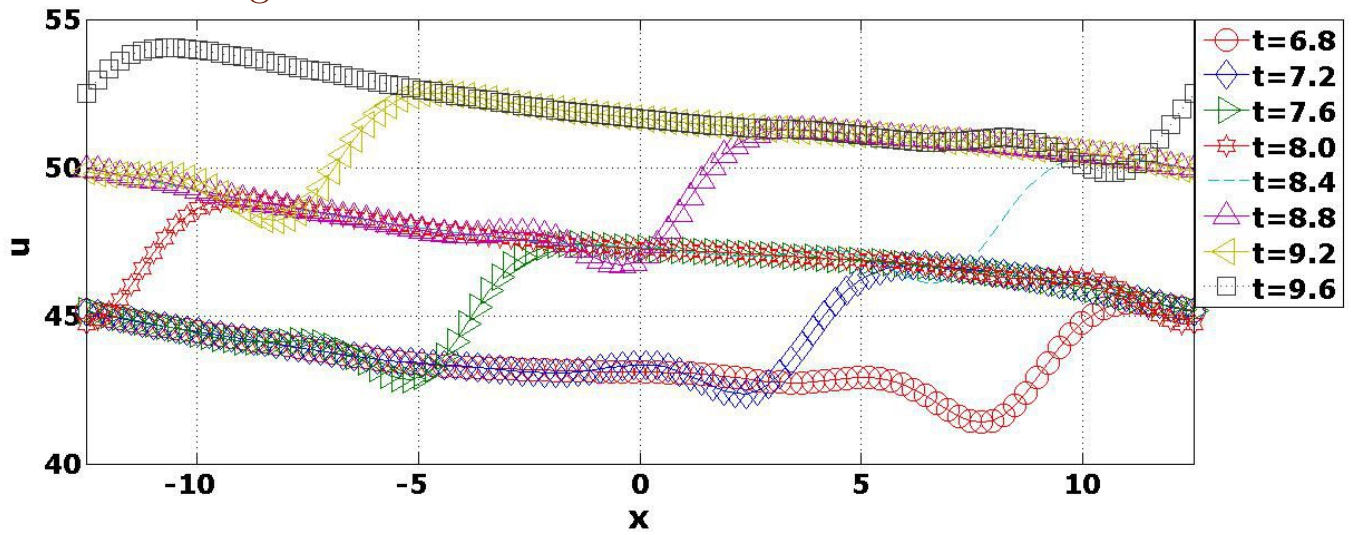


Figure 8: The surface diagram for the times between $t=0$ and $t=10$.

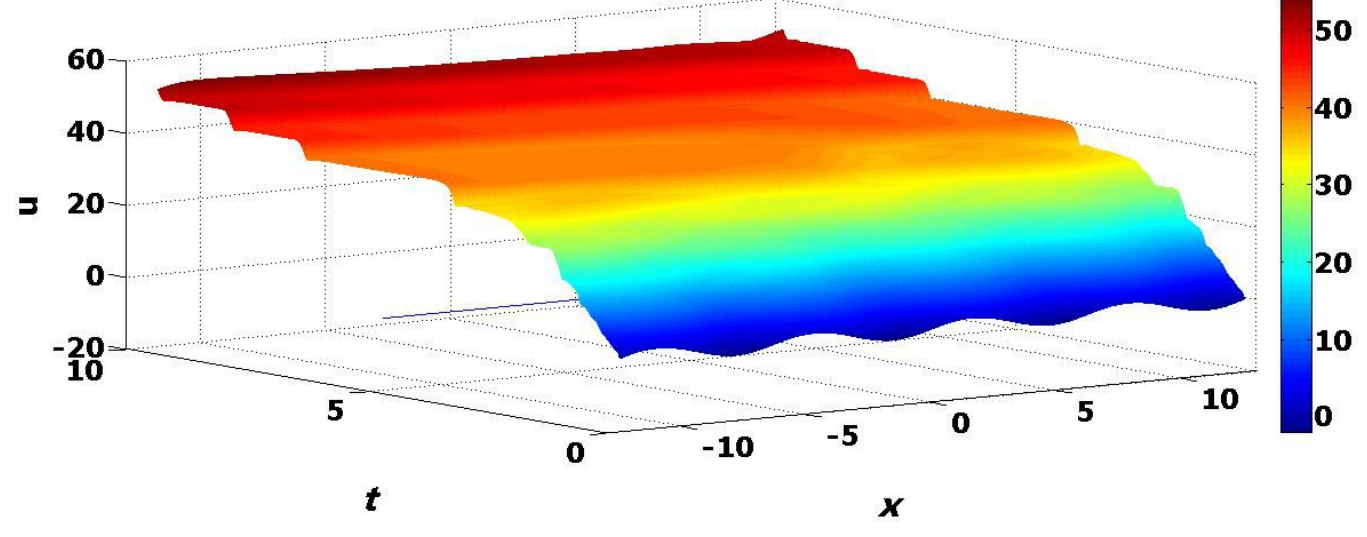

Figure 8 displays the solution dynamics in the form of a surface $u=\mathfrak{u}(t, x)$. The front's motion and shape start to settle around $t=6$, and then the velocity is practically constant. We obtained similar results when using different initial conditions and values of the parameters. Figure 9 shows the time dependence of the position of the local maximum of $\mathfrak{u}$; that is, the top point of the step. Apparently, after some transitional period characterised by fluctuations of the velocity, the graph becomes a straight line, thus indicating a constant velocity.

\section{Conclusions}

We applied the 1D-IRBF numerical method to solve equation (1), simulating spinning combustion fronts and oscillations in a certain class of reactiondiffusion systems. The method successfully reproduced the settled spinning regimes of an earlier study [1]. We also presented the complex formation process of the spinning waves, in which the energy pumping within the sharpest segments of the front overpowers the details of the initial conditions and determines the direction of motion. 
Figure 9: The propagation of the local maximum of $u$ for $t=0$ through $\mathrm{t}=10$ (top: earlier times; bottom: later times).
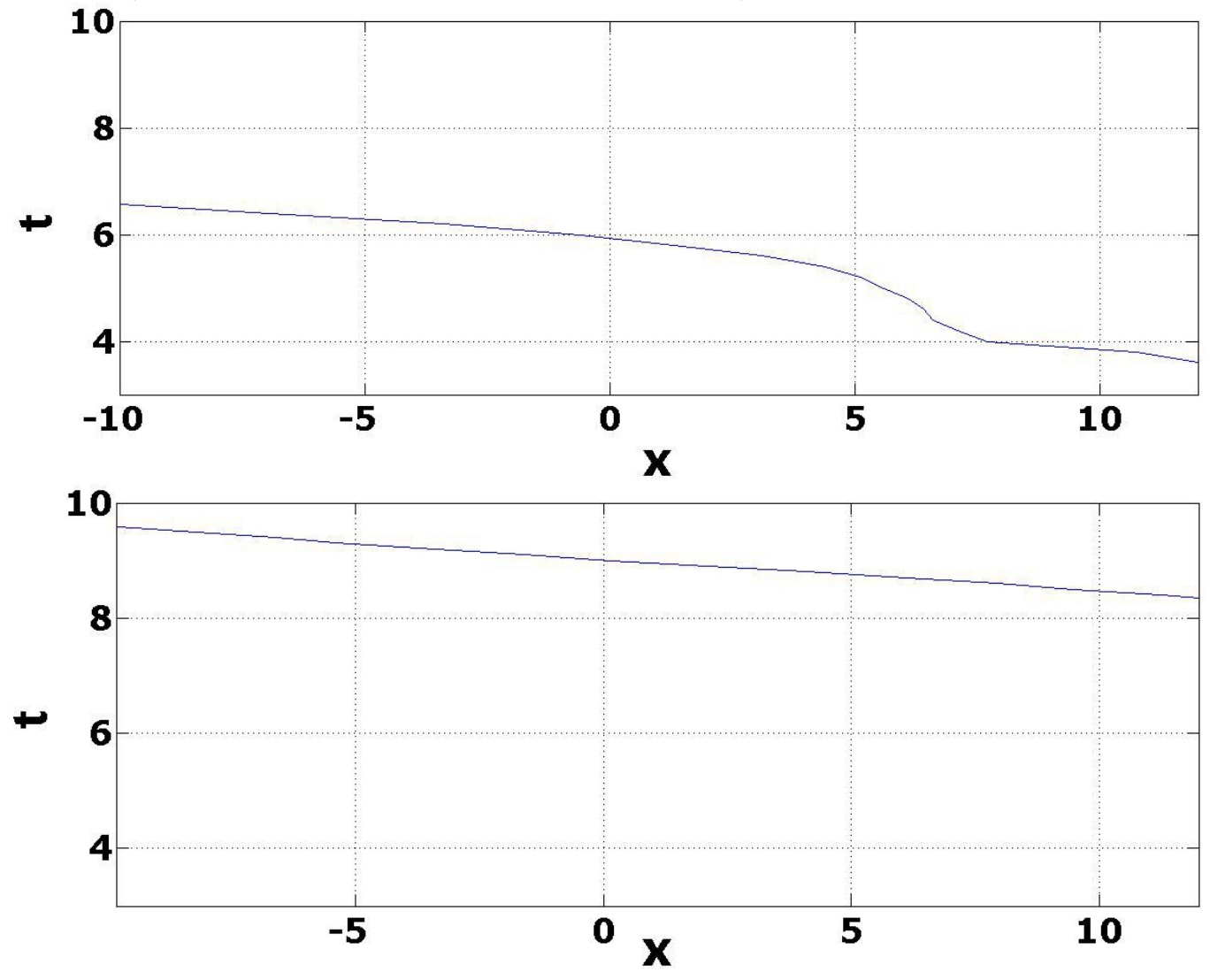


\section{References}

[1] D. V. Strunin. Autosoliton model of the spinning fronts of reaction. IMA J. Appl. Math. 63:163-177, 1999. doi:10.1093/imamat/63.2.163 C399, C400, C408

[2] D. V. Strunin. Phase equation with nonlinear excitation for nonlocally coupled oscillators. Physica D 238:1909-1916, 2009 doi:10.1016/j.physd.2009.06.022 C399, C400

[3] D. V. Strunin and M. G. Mohammed. Range of validity and intermittent dynamics of the phase of oscillators with nonlinear self-excitation. Commun. Nonlinear Sci. 29:128-147, 2015. doi:10.1016/j.cnsns.2015.04.024 C399, C400

[4] F. J. Mohammed, D. Ngo-Cong, D. V. Strunin, N. Mai-Duy and T. Tran-Cong. Modelling dispersion in laminar and turbulent flows in an open channel based on centre manifolds using 1D-IRBFN method. Appl. Math. Model. 38:3672-3691, 2014. doi:10.1016/j.apm.2013.12.007 C401

[5] N. Mai-Duy and T. Tran-Cong. Numerical solution of Navier-Stokes equations using multiquadric radial basis function networks. Int. J. Numer. Meth. Fluids 37:65-86, 2001. doi:10.1002/fld.165 C401

[6] N. Mai-Duy and R. I. Tanner. A collocation method based on one-dimensional RBF interpolation scheme for solving PDEs. Int. J. Numer. Meth. Heat Fluid 17:165-186, 2007. doi:10.1108/09615530710723948 C401

[7] D. Ngo-Cong, N. Mai-Duy, W. Karunasena and T. Tran-Cong. Local moving least square- one-dimensional IRBFN technique for incompressible viscous flows. Int. J. Numer. Meth. Fluids 70:1443-1474, 2012. doi:10.1002/fld.3640 C401

[8] D. Ngo-Cong, N. Mai-Duy, W. Karunasena and T. Tran-Cong. Free vibration analysis of laminated composite plates based on FSDT using 
one-dimensional IRBFN Method. Comput. Struct. 89:1-13, 2011. doi:10.1016/j.compstruc.2010.07.012 C401

[9] D. Ngo-Cong, N. Mai-Duy, W. Karunasena and T. Tran-Cong. A numerical procedure based on 1D-IRBFN and local MLS-1D-IRBFN methods for fluid-structure interaction analysis. Comput. Model. Eng. Sci. 83:459-498, 2012. doi:10.3970/cmes.2012.083.459 C401

[10] S. Haykin. Neural networks: A comprehensive foundation. Prentice-Hall, 1999. C401

[11] G. E. Fasshauer. Meshfree approximation methods with Matlab. Interdisciplinary mathematical Sciences, Vol. 6. World Scientific, 2007. doi:10.1142/6437 C401

[12] E. J. Kansa. Multiquadrics - a scattered data approximation scheme with applications to computational fluid-dynamics-I. Surface approximations partial derivative estimates. Comput, Math. Appl. 19:127-145, 1990. doi:10.1016/0898-1221(90)90270-T C401

[13] N. Mai-Duy and T. Tran-Cong. Numerical solution of differential equations using multiquadric radial basis function networks. Neural Net. 14:185-199, 2001. doi:10.1016/S0893-6080(00)00095-2 C400, C402

[14] N. Mai-Duy and T. Tran-Cong. Approximation of function and its derivatives using radial basis function networks. Appl. Math. Model. 27:197-220, 2003. doi:10.1016/S0307-904X(02)00101-4 C400, C402

[15] N. Mai-Duy and R. I. Tanner. Solving high order partial differential equations with radial basis function networks. Int. J. Numer. Meth. Eng. 63:1636-1654, 2005. doi:10.1002/nme.1332 C402

[16] N. Mai-Duy, H. See and T. Tran-Cong. An integral-collocation-based fictitious-domain technique for solving elliptic problems. Commun. Numer. Meth. Eng. 24:1291-1314, 2008. doi:10.1002/cnm.1033 C402 
[17] N. Mai-Duy and T. Tran-Cong. A multidomain integrated-radial-basis-function collocation method for elliptic problems. Numer. Meth. Part. D. E. 24:1301-1320, 2008. doi:10.1002/num.20319 $\mathrm{C} 402$

[18] N. Mai-Duy and T. Tran-Cong. Compact local integrated-RBF approximations for second-order elliptic differential problems. $J$. Comput. Phys. 230:4772-4794, 2011. doi:10.1016/j.jcp.2011.03.002 C402

[19] P. Le, N. Mai-Duy, T. Tran-Cong and G. Baker. A meshless modeling of dynamic strain localization in quasi-brittle materials using radial basis function networks. Comput. Model. Eng. Sci. 25:43-68, 2008. doi:10.3970/cmes.2008.025.043 C402

[20] R. Franke. Scattered data interpolation: Test of some methods. Math. Comput. 38:181-200, 1982. doi:10.1090/S0025-5718-1982-0637296-4 C401

\section{Author addresses}

1. R. P. Bhanot, Faculty of Health, Engineering and Sciences, University of Southern Queensland, Queensland 4350, Australia. orcid:0000-0002-4160-6899

2. D. V. Strunin, Faculty of Health, Engineering and Sciences, University of Southern Queensland, Queensland 4350, Australia. http://staffprofile.usq.edu.au/profile/Dmitry-Strunin mailto:strunin@usq.edu.au 